\title{
The Weighted Morrey Boundedness of Multilinear Singular Integral Operators on RD-Spaces
}

\author{
Siyuan Zhang ${ }^{1}$, Haibo $\operatorname{Lin}^{2}$ and Yan Lin ${ }^{1, *}$ \\ ${ }^{1}$ School of Science, China University of Mining and Technology, Beijing 100083, \\ China \\ ${ }^{2}$ College of Science, China Agricultural University, Beijing 100083, China
}

Received 28 September 2020; Accepted (in revised version) 21 July 2021

Dedicated to Prof. Shanzhen Lu with admiration on the occasion of his 80th birthday

\begin{abstract}
An RD-space $\mathcal{X}$ is a space of homogeneous type in the sense of Coifman and Weiss, which is equipped with a measure satisfying an additional reverse doubling property. In this paper we study the boundedness of multilinear singular integral operators in weighted Morrey spaces within the framework of RD-spaces.
\end{abstract}

Key Words: RD-space, multilinear Calderón-Zygmund operator, multiple weight, weighted Morrey space.

AMS Subject Classifications: 43A85, 47H60

\section{Introduction}

The Calderón-Zygmund singular integral operator is a natural generalization of the Hilbert transform and the Riesz transform on the Euclidean space, and its corresponding theories have been proven to be a powerful tool in many aspects of harmonic analysis and partial differential equations. The exploration of multilinear operators is not only motivated by the generalization of linear operators, but also their natural appearance in analysis. The study on the class of multilinear Calderón-Zygmund operators was preliminarily considered by Coifman and Meyer in [4] and was systematically discussed by Grafakos and Torres in [10]. Since then, a lot of papers focus on this field of research. The following are some recent development: Grafakos et al. [8] provided a self-contained proof of the multilinear extension of the Marcinkiewicz real method interpolation theorem; Mo and Lu [18] established the boundedness of commutators generated by the multilinear Calderón-Zygmund type singular integrals and Lipschitz functions on the

*Corresponding author. Email addresses: zsy494355731@163.com (S. Zhang), haibolincau@126.com (H. Lin), linyan@cumtb.edu.cn (Y. Lin) 
Triebel-Lizorkin space and Lipschitz spaces; Lin et al. [17] obtained some sharp maximal estimates for multilinear commutators of multilinear strongly singular CalderónZygmund operators.

On the other hand, the weighted inequalities arise naturally in Fourier analysis, but their use is best justified by the variety of applications in which they appear; see, for instance, Grafakos [7]. Grafakos and Torres [11] established the weighted estimates with $A_{p}$ weights for the multilinear Calderón-Zygmund operator and the corresponding maximal operator. The corresponding results are generalized to the multilinear singular integral operators with non-smooth kernels by $\mathrm{Hu}$ and $\mathrm{Lu}$ [13]. In 2009, Lerner et al. [16] developed the theory of the multiple $A_{p}$ weight, which was applied to the weighted estimates for multilinear Calderón-Zygmund operators. Recently, Chen et al. [1] established the multiple weighted norm inequalities for the maximal vector-valued multilinear Calderón-Zygmund operators. In 2013, Wang and Yi [22] studied the boundedness properties of multilinear Calderón-Zygmund operators on products of weighted Morrey spaces with multiple weights. We mention that the classical Morrey spaces were introduced by Morrey [19] in order to study the existence and differentiability properties of the solutions of second-order elliptic partial differential equations, and the weighted Morrey space was first introduced by Komori and Shirai [15].

With the deepening of theoretical research, it has been discovered that a number of remarkable problems do not need to be embedded in the framework of Euclidean spaces equipped with Lebesgue measures. A prime example is the spaces of homogeneous type introduced by Coifman and Weiss $[5,6]$. In the past decade, the analysis on RD-space, which is a special space of homogeneous type equipped with measures satisfying an additional reverse doubling property, has been greatly developed; see, for instance, [3,9, $12,14,20,23-25]$ and the references therein. It should be pointed out that the theory of multilinear operators on RD-spaces has been discussed by Grafakos et al. [9] in a unified way. Moreover, Kokilashvili and Meskhi [14] introduced the weighted Morrey space on RD-spaces.

Inspired by the above works, the main purpose of this paper is to establish the boundedness properties of multilinear Caldeŕon-Zygmund operators on products of weighted Morrey spaces with multiple weights defined on RD-spaces.

To illustrate our main results, we first review some necessary definitions and notation. We begin with the definition of RD-space.

Definition 1.1. Let $(\mathcal{X}, d)$ be a metric space and for any $x \in \mathcal{X}$ and $r \in(0, \infty)$, let the ball $B(x, r):=\{y \in \mathcal{X}: d(x, y)<r\}$. Suppose that $\mu$ is a regular Borel measure defined on a $\sigma$-algebra which contains all Borel sets induced by the open balls $\{B(x, r): x \in \mathcal{X}, r>0\}$, and that $0<\mu(B(x, r))<\infty$ for all $x \in \mathcal{X}$ and $r \in(0, \infty)$.

(i) The triple $(\mathcal{X}, d, \mu)$ is called a space of homogeneous type if there exists a constant $C_{1} \in$ $[1, \infty)$ such that, for any $x \in \mathcal{X}$ and $r \in(0, \infty)$,

$$
\mu(B(x, 2 r)) \leq C_{1} \mu(B(x, r)) .
$$


(ii) The triple $(\mathcal{X}, d, \mu)$ is called an $R D$-space if there exist constants $\tau \in(0, n]$ and $C_{2} \in$ $[1, \infty)$ such that, for any $x \in \mathcal{X}, r \in(0, \operatorname{diam}(\mathcal{X}) / 2)$ and $\lambda \in[1, \operatorname{diam}(X) /(2 r))$,

$$
\left(C_{2}\right)^{-1} \lambda^{\tau} \mu(B(x, r)) \leq \mu(B(x, \lambda r)) \leq C_{2} \lambda^{n} \mu(B(x, r)),
$$

where

$$
\operatorname{diam}(\mathcal{X}):=\sup _{x, y \in \mathcal{X}} d(x, y)
$$

Remark 1.1. (i) Obviously, an RD-space is a space of homogeneous.

(ii) It has been shown in [12] that, if $\mu$ satisfies (1.1), then $\mu$ satisfies (1.2) if and only if there exist constants $a_{0}, C_{0} \in(1, \infty)$ such that, for any $x \in \mathcal{X}$ and $r \in\left(0, \operatorname{diam}(\mathcal{X}) / a_{0}\right)$,

$$
\mu\left(B\left(x, a_{0} r\right)\right) \geq C_{0} \mu(B(x, r)),
$$

and equivalently for any $x \in \mathcal{X}$ and $r \in\left(0, \operatorname{diam}(\mathcal{X}) / a_{0}\right)$,

$$
B\left(x, a_{0} r\right) \backslash B(x, r) \neq \varnothing,
$$

which is known in the topology as the uniform prefectness. For more details, see also [24].

Throughout the whole paper, we always assume that the underlying space $(\mathcal{X}, d, \mu)$ is an RD-space with $\mu(\mathcal{X})=\infty$. Motivated by [9] we study the following multilinear singular integrals on $(\mathcal{X}, d, \mu)$.

Definition 1.2. For $\eta \in(0,1]$, let $C^{\eta}(\mathcal{X})$ be the set of all functions $f: \mathcal{X} \rightarrow \mathbb{C}$ such that

$$
\|f\|_{C^{\eta}(\mathcal{X})}:=\sup _{x \neq y} \frac{|f(x)-f(y)|}{d(x, y)^{\eta}}<\infty .
$$

Denote by $\operatorname{supp}(f)$ the closure of the set $\{x \in \mathcal{X}: f(x) \neq 0\}$ in $\mathcal{X}$. Define

$$
C_{b}^{\eta}(\mathcal{X}):=\left\{f \in C^{\eta}(\mathcal{X}): f \text { has bounded support }\right\} .
$$

Denote by $\left(C_{b}^{\eta}(\mathcal{X})\right)^{\prime}$ the dual space of $C_{b}^{\eta}(\mathcal{X})$, namely, the collection of all continuous linear functionals on $C_{b}^{\eta}(\mathcal{X})$.

Given $m \in \mathbb{N}$, set

$$
\begin{aligned}
& \Omega_{m}:=\mathcal{X}^{m+1} \backslash\left\{\left(y_{0}, y_{1}, \cdots, y_{m}\right): y_{0}=y_{1}=\cdots=y_{m}\right\} \\
& V(x, y):=\mu(B(x, d(x, y))) \text { for all } x, y \in \mathcal{X} .
\end{aligned}
$$

Definition 1.3. Suppose that $K: \Omega_{m} \rightarrow \mathbb{C}$ is locally integrable. The function $K$ is called $a$ Calderón-Zygmund kernel if there exist constants $C_{K} \in(0, \infty)$ and $\gamma \in(0,1]$ such that, for all $\left(y_{0}, y_{1}, \cdots, y_{m}\right) \in \Omega_{m}$,

$$
\left|K\left(y_{0}, y_{1}, \cdots, y_{m}\right)\right| \leq C_{K}\left[\sum_{j=1}^{m} V\left(y_{0}, y_{k}\right)\right]^{-m},
$$


and that, for all $k \in\{0,1, \cdots, m\}$,

$$
\begin{aligned}
& \left|K\left(y_{0}, y_{1}, \cdots, y_{k}, \cdots, y_{m}\right)-K\left(y_{0}, y_{1}, \cdots, y_{k}^{\prime}, \cdots, y_{m}\right)\right| \\
\leq & C_{K}\left[\frac{d\left(y_{k}, y_{k}^{\prime}\right)}{\max _{0 \leq k \leq m} d\left(y_{0}, y_{k}\right)}\right]^{\gamma}\left[\sum_{k=1}^{m} V\left(y_{0}, y_{k}\right)\right]^{-m},
\end{aligned}
$$

whenever

$$
d\left(y_{k}, y_{k}^{\prime}\right) \leq \frac{1}{2} \max _{0 \leq k \leq m} d\left(y_{0}, y_{k}\right)
$$

In this case, we write $K \in \operatorname{Ker}\left(m, C_{K}, \gamma\right)$.

Definition 1.4. Let $\eta \in(0,1]$. An m-linear Calderón-Zygmund operator is a continuous operator

$$
T: \overbrace{C_{b}^{\eta}(\mathcal{X}) \times \cdots \times C_{b}^{\eta}(\mathcal{X})}^{\text {mtimes }} \rightarrow\left(C_{b}^{\eta}(\mathcal{X})\right)^{\prime}
$$

such that, for all $f_{1}, \cdots, f_{m} \in C_{b}^{\eta}(\mathcal{X})$ and $x \notin \cap_{i=1}^{m} \operatorname{supp}\left(f_{i}\right)$,

$$
T\left(f_{1}, \cdots, f_{m}\right)(x)=\int_{\mathcal{X}^{m}} K\left(x, y_{1}, \cdots, y_{m}\right) \prod_{i=1}^{m} f_{i}\left(y_{i}\right) d \mu\left(y_{1}\right) \cdots d \mu\left(y_{m}\right),
$$

where the kernel $K \in \operatorname{Ker}\left(m, C_{K}, \gamma\right)$ for some $C_{K} \in(0, \infty)$ and $\gamma \in(0,1]$.

In what follows, for any $p \in[1, \infty)$, let $p^{\prime}:=p /(p-1)$ denote its conjugate index. Given a measure $\rho$ absolutely continuous with respect to the measure $\mu$, that is, there is a non-negative locally integrable function $\omega$ such that $d \rho(x)=\omega(x) d \mu(x)$ for all $x \in \mathcal{X}$, then $\rho$ is called a weighted measure with respect to $\mu$ and $\omega$ is called a weight. A weight $\omega$ is said to belong to the Muckenhoupt class $A_{p}(\mu)$ for $p \in(1, \infty)$ if

$$
[\omega]_{A_{p}}:=\sup _{B}\left[\frac{1}{\mu(B)} \int_{B} \omega(y) d \mu(y)\right]\left[\frac{1}{\mu(B)} \int_{B} \omega(y)^{1-p^{\prime}} d \mu(y)\right]^{p-1}<\infty,
$$

where the supremum is taken over all balls $B$ contained in $\mathcal{X}$. When $p=1$, a weight $\omega$ is said to belong to the Muckenhoupt class $A_{1}(\mu)$ if

$$
[\omega]_{A_{1}}:=\sup _{B}\left[\frac{1}{\mu(B)} \int_{B} \omega(y) d \mu(y)\right]\left[\inf _{B} \omega(x)\right]^{-1}<\infty .
$$

Set

$$
A_{\infty}(\mu):=\bigcup_{1 \leq p<\infty} A_{p}(\mu)
$$

For $m$ exponents $p_{1}, \cdots, p_{m}$, write $p$ for the exponent defined by

$$
\frac{1}{p}=\frac{1}{p_{1}}+\cdots+\frac{1}{p_{m}}
$$

and $\vec{P}:=\left(p_{1}, \cdots, p_{m}\right)$. 
Definition 1.5. Let $p_{1}, \cdots, p_{m} \in[1, \infty)$ and $p \in(0, \infty)$ such that

$$
\frac{1}{p}=\sum_{j=1}^{m} \frac{1}{p_{j}}
$$

Set

$$
\vec{\omega}:=\left(\omega_{1}, \cdots, \omega_{m}\right) \quad \text { and } \quad v_{\vec{\omega}}:=\prod_{i=1}^{m} \omega_{i}^{p / p_{i}}
$$

for every $\omega_{j}$ being a weight. We say that $\vec{\omega}$ satisfies the $A_{\vec{P}}$ condition if

$$
\sup _{B}\left[\frac{1}{\mu(B)} \int_{B} v_{\vec{\omega}}(x) d \mu(x)\right]^{1 / p} \prod_{j=1}^{m}\left[\frac{1}{\mu(B)} \int_{B} \omega_{j}(x)^{1-p_{j}^{\prime}} d \mu(x)\right]^{1 / p_{j}^{\prime}}<\infty,
$$

where, when $p_{j}=1$,

$$
\left[\frac{1}{\mu(B)} \int_{B} \omega_{j}(x)^{1-p_{j}^{\prime}} d \mu(x)\right]^{1 / p_{j}^{\prime}}
$$

is understood as $\inf _{B} \omega_{j}^{-1}$.

In the remainder of this article, for any measurable set $E$ and a weight $\omega$, we set

$$
\omega(E):=\int_{E} \omega(x) d \mu(x) .
$$

Definition 1.6. Let $p \in(0, \infty)$ and $\kappa \in(0,1)$, and let $\omega$ be a weight. Then the weighted Morrey space is defined by setting

$$
M_{\omega}^{p, \kappa}(\mathcal{X}):=\left\{f \in L_{l o c}^{p}(\omega):\|f\|_{M_{\omega}^{p, \kappa}(\mathcal{X})}<\infty\right\},
$$

where

$$
\|f\|_{M_{\omega}^{p, \kappa}(\mathcal{X})}:=\sup _{B}\left[\frac{1}{[\omega(B)]^{\kappa}} \int_{B}|f(x)|^{p} \omega(x) d \mu(x)\right]^{\frac{1}{p}} .
$$

We also denote by $\mathrm{WM}_{\omega}^{p, \kappa}(\mathcal{X})$ the generalized weighted weak Morrey space of all locally integrable functions satisfying

$$
\|f\|_{W M_{\omega}^{p, \kappa}(\mathcal{X})}:=\sup _{B} \sup _{t>0} \frac{1}{[\omega(B)]^{\kappa / p}} t \omega(\{x \in B:|f(x)|>t\})^{\frac{1}{p}}<\infty .
$$

Remark 1.2. If $\kappa \equiv 0, M_{\omega}^{p, \kappa}(\mathcal{X})$ is just the weighted Lebesgue spaces.

We now formulate our main results as follows. 
Theorem 1.1. Let $m \geq 2$, and $T$ be an m-linear Calderón-Zygmund operator. If $p_{1}, \cdots, p_{m} \in(1, \infty)$ with $\frac{1}{p}=\frac{1}{p_{1}}+\cdots+\frac{1}{p_{m}}$ and $\vec{\omega}=\left(\omega_{1}, \cdots, \omega_{m}\right) \in A_{\vec{p}}$ with $\omega_{1}, \cdots, \omega_{m} \in A_{\infty}(\mu)$, then for any $\kappa \in(0,1)$, there exists a positive constant $C$ independent of $\vec{f}=\left(f_{1}, \cdots, f_{m}\right)$ such that

$$
\|T(\vec{f})\|_{M_{v_{\vec{\omega}}}^{p, \kappa}(\mathcal{X})} \leq C \prod_{i=1}^{m}\left\|f_{i}\right\|_{M_{\omega_{i}}^{p_{i}, \kappa}(\mathcal{X})^{\prime}}
$$

where

$$
v_{\vec{\omega}}=\prod_{i=1}^{m} \omega_{i}^{p / p_{i}}
$$

Theorem 1.2. Let $m \geq 2$, and $T$ be an m-linear Calderón-Zygmund operator. If $p_{1}, \cdots, p_{m} \in$ $[1, \infty)$ with some $p_{i}=1$ and $\frac{1}{p}=\frac{1}{p_{1}}+\cdots+\frac{1}{p_{m}}$ and $\vec{\omega}=\left(\omega_{1}, \cdots, \omega_{m}\right) \in A_{\vec{p}}$ with $\omega_{1}, \cdots, \omega_{m} \in A_{\infty}(\mu)$, then for any $\kappa \in(0,1)$, there exists a positive constant $C$ independent of $\vec{f}=\left(f_{1}, \cdots, f_{m}\right)$ such that

$$
\|T(\vec{f})\|_{W M_{v}^{p, \kappa}(\mathcal{X})} \leq C \prod_{i=1}^{m}\left\|f_{i}\right\|_{M_{\omega_{i}}^{p_{i}, \kappa}(\mathcal{X})^{\prime}}
$$

where $v_{\vec{\omega}}$ is given by (1.8).

This paper is organized as follows. In Section 2, we recall some useful lemmas associated with the weights as well as the weighted $L^{p}$-boundedness of the $m$-linear CalderónZygmund operator. Section 3 is devoted to prove Theorems 1.1 and 1.2.

Finally, we make some conventions on notation. Throughout this paper, we always denote by $C$ a positive constant which is independent of the main parameters, but they may vary from line to line. Constants with subscripts, such as $C_{1}$ and $C_{2}$, do not change in different occurrences. The expression $Y \lesssim Z$ means that there exists a positive constant $C$ such that $Y \leq C Z$. The expression $A \sim B$ means that $A \lesssim B \lesssim A$. Also, for any subset $E \subset \mathcal{X}, \chi_{E}$ denotes its characteristic function.

\section{Preliminaries}

In this section, we show several lemmas which will be used in the proof of Theorems 1.1 and 1.2. We begin with the properties of the $A_{p}$ and $A_{\vec{p}}$ weights.

Lemma 2.1 ([21, Theorem 15]). Let $\omega \in A_{p}(\mu)$ with $p \in[1, \infty)$. Then there exist positive constants $C_{3}$ and $C_{4}$ such that, for any ball $B \subset \mathcal{X}$ and each measurable set $E \subset B$,

$$
\begin{aligned}
& \frac{\omega(E)}{\omega(B)} \leq C_{3}\left[\frac{\mu(E)}{\mu(B)}\right]^{\frac{1}{p}}, \\
& \frac{\omega(E)}{\omega(B)} \geq C_{4}\left[\frac{\mu(E)}{\mu(B)}\right]^{p} .
\end{aligned}
$$


Lemma 2.2 ([9]). Let $p_{1}, \cdots, p_{m} \in[1, \infty)$, and

$$
\frac{1}{p}=\sum_{k=1}^{m} \frac{1}{p_{k}}
$$

Then $\vec{\omega}=\left(\omega_{1}, \cdots, \omega_{m}\right) \in A_{\vec{p}}$ if and only if

$$
\left\{\begin{array}{l}
\omega_{j}^{1-p_{j}^{\prime}} \in A_{m p_{j}^{\prime},} \quad j=1, \cdots, m, \\
v_{\vec{\omega}} \in A_{m p}
\end{array}\right.
$$

where $v_{\vec{\omega}}$ is given by (1.8), and the condition $\omega_{j}^{1-p_{j}^{\prime}} \in A_{m p_{j}^{\prime}}$ in the case $p_{j}=1$ is understood as $\omega_{j}^{1 / m} \in A_{1}$.

Lemma 2.3 ( $[2$, p. 60]). Let $(\Omega, d, \mu)$ be a nonnegative metric measure space with $\mu(\Omega)<\infty$. Let $f \in L^{1}(\Omega)$ and $f(\Omega) \subset I$ with I being an interval. If $\Phi$ is convex on I, then the following Jensen inequality

$$
\Phi\left(\frac{1}{\mu(\Omega)} \int_{\Omega} f d \mu\right) \leq \frac{1}{\mu(\Omega)} \int_{\Omega} \Phi \circ f d \mu
$$

holds, provided that $\Phi \circ f \in L^{1}(\Omega)$.

Lemma 2.4. Let $\omega \in A_{\infty}(\mu)$, then for all balls $B \subset \mathcal{X}$, the following reverse Jensen inequality holds:

$$
\frac{1}{\mu(B)} \int_{B} \omega(x) d \mu(x) \leq C \exp \left\{\frac{1}{\mu(B)} \int_{B} \log \omega(x) d \mu(x)\right\},
$$

where $C$ is a positive constant independent of $B$.

Proof. If $\omega \in A_{\infty}(\mu)$, then there exists some $p \in(1, \infty)$ such that $\omega \in A_{p}(\mu)$. On the other hand, notice that $e^{x}$ is convex on $(-\infty, \infty)$, if we replace $f$ by $\log \left[\left(\frac{1}{\omega(x)}\right)^{1 /(p-1)}\right]$ in Lemma 2.3, it is easy to see that

$$
\frac{1}{\mu(B)} \int_{B} \log \left[\left(\frac{1}{\omega(x)}\right)^{1 /(p-1)}\right] d \mu(x) \leq \log \left\{\frac{1}{\mu(B)} \int_{B}\left[\left(\frac{1}{\omega(x)}\right)^{1 /(p-1)}\right] d \mu(x)\right\},
$$

which is equivalent to

$$
\exp \left\{\frac{1}{\mu(B)} \int_{B} \log \frac{1}{\omega(x)} d \mu(x)\right\} \leq\left[\frac{1}{\mu(B)} \int_{B}\left(\frac{1}{\omega(x)}\right)^{1 /(p-1)} d \mu(x)\right]^{p-1} .
$$

This, together with (1.5), implies that (2.1) holds true. 
Lemma 2.5. Let $m \geq 2, p_{1}, \cdots, p_{m} \in[1, \infty)$, and $p \in(0, \infty)$ with $1 / p=\sum_{k=1}^{m} 1 / p_{k}$. Assume that $\omega_{1}, \cdots, \omega_{m} \in A_{\infty}(\mu)$ and $v_{\vec{\omega}}$ is given by (1.8), then there exists a positive constant $C$ such that, for any ball $B$,

$$
\prod_{i=1}^{m}\left(\int_{B} \omega_{i}(x) d \mu(x)\right)^{p / p_{i}} \leq C \int_{B} v_{\vec{\omega}}(x) d \mu(x) .
$$

Proof. Since $\omega_{1}, \cdots, \omega_{m} \in A_{\infty}(\mu)$, then by using Lemma 2.4 , we have

$$
\begin{aligned}
\prod_{i=1}^{m}\left(\int_{B} \omega_{i}(x) d \mu(x)\right)^{p / p_{i}} & \lesssim \prod_{i=1}^{m}\left(\mu(B) \cdot \exp \left\{\frac{1}{\mu(B)} \int_{B} \log \omega_{i}(x) d \mu(x)\right\}\right)^{p / p_{i}} \\
& =\prod_{i=1}^{m}\left(\mu(B)^{p / p_{i}} \cdot \exp \left\{\frac{1}{\mu(B)} \int_{B} \log \left[\omega_{i}(x)\right]^{p / p_{i}} d \mu(x)\right\}\right) \\
& =(\mu(B))^{\sum_{i=1}^{m} p / p_{i}} \cdot \exp \left\{\sum_{i=1}^{m} \frac{1}{\mu(B)} \int_{B} \log \left[\omega_{i}(x)\right]^{p / p_{i}} d \mu(x)\right\}
\end{aligned}
$$

Notice that

$$
\sum_{i=1}^{m} p / p_{i}=1 \quad \text { and } \quad v_{\vec{\omega}}(x)=\prod_{i=1}^{m} \omega_{i}(x)^{p / p_{i}}
$$

It then follow from Lemma 2.3 that

$$
\begin{aligned}
\prod_{i=1}^{m}\left(\int_{B} \omega_{i}(x) d \mu(x)\right)^{p / p_{i}} & \lesssim \mu(B) \exp \left[\frac{1}{\mu(B)} \int_{B} \log \left(\prod_{i=1}^{m}\left(\omega_{i}(x)\right)^{p / p_{i}}\right) d \mu(x)\right] \\
& \lesssim \int_{B} v_{\vec{\omega}}(x) d \mu(x)
\end{aligned}
$$

which completes the proof of Lemma 2.5 .

Grafakos et al. [9] gave the following weighted $L^{p}$-boundedness of multilinear Caldeŕon-Zygmund operators on RD-spaces.

Lemma 2.6 ([9]). Let $p_{1}, \cdots, p_{m} \in[1, \infty), \frac{1}{p}=\frac{1}{p_{1}}+\cdots+\frac{1}{p_{m}}, \vec{P}:=\left(p_{1}, \cdots, p_{m}\right)$, and $\vec{\omega}=\left(\omega_{1}, \cdots, \omega_{m}\right) \in A_{\vec{p}}$. Then the following hold:

(i) $T$ can be extended to a bounded m-linear operator from $L^{p_{1}}\left(\omega_{1}\right) \times \cdots \times L^{p_{m}}\left(\omega_{m}\right)$ to $L^{p}\left(v_{\vec{\omega}}\right)$ if all the exponents $p_{j}$ are greater than 1 ;

(ii) $T$ can be extended to a bounded m-linear operator from $L^{p_{1}}\left(\omega_{1}\right) \times \cdots \times L^{p_{m}}\left(\omega_{m}\right)$ to $L^{p, \infty}\left(v_{\vec{\omega}}\right)$ if some of the exponents $p_{j}$ are equal to 1 . 


\section{Proof of Theorems 1.1 and 1.2}

For the sake of brevity, we will limit our proof to the case of $m=2$.

Proof of Theorem 1.1. For any ball $B=B\left(c_{B}, r_{B}\right) \subset \mathcal{X}$, decompose $f_{i}=f_{i}^{0}+f_{i}^{\infty}$, where $f_{i}^{0}=f_{i} \chi_{2 B}, i=1,2$. Then we have

$$
\begin{aligned}
\prod_{i=1}^{2} f_{i}\left(y_{i}\right) & =\prod_{i=1}^{2}\left(f_{i}^{0}\left(y_{i}\right)+f_{i}^{\infty}\left(y_{i}\right)\right) \\
& =f_{1}^{0}\left(y_{1}\right) f_{2}^{0}\left(y_{2}\right)+f_{1}^{0}\left(y_{1}\right) f_{2}^{\infty}\left(y_{2}\right)+f_{1}^{\infty}\left(y_{1}\right) f_{2}^{0}\left(y_{2}\right)+f_{1}^{\infty}\left(y_{1}\right) f_{2}^{\infty}\left(y_{2}\right),
\end{aligned}
$$

which, together with the fact that $T$ is an multilinear operator, shows that

$$
\begin{aligned}
& \quad \frac{1}{\left[v_{\vec{\omega}}(B)\right]^{\kappa / p}}\left(\int_{B}\left|T\left(f_{1}, f_{2}\right)(x)\right|^{p} v_{\vec{\omega}}(x) d \mu(x)\right)^{1 / p} \\
& \lesssim \frac{1}{\left[v_{\vec{\omega}}(B)\right]^{\kappa / p}}\left(\int_{B}\left|T\left(f_{1}^{0}, f_{2}^{0}\right)(x)\right|^{p} v_{\vec{\omega}}(x) d \mu(x)\right)^{1 / p} \\
& \quad+\frac{1}{\left[v_{\vec{\omega}}(B)\right]^{\kappa / p}}\left(\int_{B}\left|T\left(f_{1}^{0}, f_{2}^{\infty}\right)(x)\right|^{p} v_{\vec{\omega}}(x) d \mu(x)\right)^{1 / p} \\
& \quad+\frac{1}{\left[v_{\vec{\omega}}(B)\right]^{\kappa / p}}\left(\int_{B}\left|T\left(f_{1}^{\infty}, f_{2}^{0}\right)(x)\right|^{p} v_{\vec{\omega}}(x) d \mu(x)\right)^{1 / p} \\
& \quad+\frac{1}{\left[v_{\vec{\omega}}(B)\right]^{\kappa / p}}\left(\int_{B}\left|T\left(f_{1}^{\infty}, f_{2}^{\infty}\right)(x)\right|^{p} v_{\vec{\omega}}(x) d \mu(x)\right)^{1 / p} \\
& =: I_{1}+I_{2}+I_{3}+I_{4} .
\end{aligned}
$$

In view of Lemma 2.2, we have that $v_{\vec{\omega}} \in A_{2 p}(\mu)$. Applying Lemmas 2.6, 2.5 and 2.1 and (1.2), we get

$$
\begin{aligned}
I_{1} & \leq \frac{1}{\left[v_{\vec{\omega}}(B)\right]^{\kappa / p}}\left(\int_{\mathcal{X}}\left|T\left(f_{1}^{0}, f_{2}^{0}\right)(x)\right|^{p} v_{\vec{\omega}}(x) d \mu(x)\right)^{1 / p} \\
& \lesssim \frac{1}{\left[v_{\vec{\omega}}(B)\right]^{\kappa / p}} \prod_{i=1}^{2}\left(\int_{\mathcal{X}}\left|f_{i}^{0}(x)\right|^{p_{i}} \omega_{i}(x) d \mu(x)\right)^{1 / p_{i}} \\
& =\frac{1}{\left[v_{\vec{\omega}}(B)\right]^{\kappa / p}} \prod_{i=1}^{2}\left(\int_{2 B}\left|f_{i}(x)\right|^{p_{i}} \omega_{i}(x) d \mu(x)\right)^{1 / p_{i}} \\
& =\prod_{i=1}^{2}\left[\frac{1}{\left[\omega_{i}(2 B)\right]^{\kappa}}\left(\int_{2 B}\left|f_{i}(x)\right|^{p_{i}} \omega_{i}(x) d \mu(x)\right)\right]^{1 / p_{i}} \cdot \prod_{i=1}^{2}\left[\omega_{i}(2 B)\right]^{\kappa / p_{i}} \cdot\left[v_{\vec{\omega}}(B)\right]^{-\kappa / p} \\
& \leq \prod_{i=1}^{2}\left\|f_{i}\right\|_{M_{\omega_{i}}^{p_{i}, \kappa}(\mathcal{X})} \cdot \prod_{i=1}^{2}\left[\omega_{i}(2 B)\right]^{\kappa / p_{i}} \cdot\left[v_{\vec{\omega}}(B)\right]^{-\kappa / p}
\end{aligned}
$$




$$
\begin{aligned}
& =\prod_{i=1}^{2}\left\|f_{i}\right\|_{M_{\omega_{i}}^{p_{i}, \kappa}(\mathcal{X})} \cdot\left[\prod_{i=1}^{2}\left(\int_{2 B} \omega_{i}(x) d \mu(x)\right)^{p / p_{i}}\right]^{\kappa / p} \cdot\left[v_{\vec{\omega}}(B)\right]^{-\kappa / p} \\
& \lesssim \prod_{i=1}^{2}\left\|f_{i}\right\|_{M_{\omega_{i}}^{p_{i}, \kappa}(\mathcal{X})} \cdot \frac{\left[v_{\vec{\omega}}(2 B)\right]^{\kappa / p}}{\left[v_{\vec{\omega}}(B)\right]^{\kappa / p}} \\
& \lesssim \prod_{i=1}^{2}\left\|f_{i}\right\|_{M_{\omega_{i}}^{p_{i}, \kappa}(\mathcal{X})}
\end{aligned}
$$

For the other terms, let us first consider the $I_{4}$. Notice that, for any $x \in B$ and $y \in$ $\mathcal{X} \backslash(2 B)$, we have $V(x, y) \sim V\left(c_{B}, y\right)$. It then follows from (1.3) and (1.2) that

$$
\begin{aligned}
\left|T\left(f_{1}^{\infty}, f_{2}^{\infty}\right)(x)\right| & \lesssim \int_{(\mathcal{X} \backslash 2 B)^{2}}\left|K\left(x, y_{1}, y_{2}\right)\right|\left|\prod_{i=1}^{2} f_{i}\left(y_{i}\right)\right| d \mu\left(y_{1}\right) d \mu\left(y_{2}\right) \\
& \lesssim \int_{(\mathcal{X} \backslash 2 B)^{2}} \prod_{i=1}^{2}\left|f_{i}\left(y_{i}\right)\right|\left[\sum_{i=1}^{2} V\left(x, y_{i}\right)\right]^{-2} d \mu\left(y_{1}\right) d \mu\left(y_{2}\right) \\
& \lesssim \int_{(\mathcal{X} \backslash 2 B)^{2}} \prod_{i=1}^{2}\left|f_{i}\left(y_{i}\right)\right|\left[\sum_{i=1}^{2} V\left(c_{B}, y_{i}\right)\right]^{-2} d \mu\left(y_{1}\right) d \mu\left(y_{2}\right) \\
& \lesssim \int_{(\mathcal{X})^{2} \backslash(2 B)^{2}} \prod_{i=1}^{2}\left|f_{i}\left(y_{i}\right)\right|\left[\sum_{i=1}^{2} V\left(c_{B}, y_{i}\right)\right]^{-2} d \mu\left(y_{1}\right) d \mu\left(y_{2}\right) \\
& \lesssim \sum_{j=1}^{\infty} \int_{\left(2^{j+1} B\right)^{2} \backslash\left(2^{j} B\right)^{2}} \prod_{i=1}^{2}\left|f_{i}\left(y_{i}\right)\right|\left[\sum_{i=1}^{2} V\left(c_{B}, y_{i}\right)\right]^{-2} d \mu\left(y_{1}\right) d \mu\left(y_{2}\right) \\
& \lesssim \sum_{j=1}^{\infty} \frac{1}{\left[\mu\left(\frac{2^{j} B}{\sqrt{2}}\right)\right]^{2}} \int_{\left(2^{j+1} B\right)^{2} \backslash\left(2^{j} B\right)^{2}} \prod_{i=1}^{2}\left|f_{i}\left(y_{i}\right)\right| d \mu\left(y_{1}\right) d \mu\left(y_{2}\right) \\
& \lesssim \sum_{j=1}^{\infty} \prod_{i=1}^{2} \frac{\mu\left(2^{j+1} B\right)}{\mu\left(2^{j} B\right)} \cdot \frac{1}{\mu\left(2^{j+1} B\right)} \int_{2^{j+1} B}\left|f_{i}\left(y_{i}\right)\right| d \mu\left(y_{i}\right) \\
& \lesssim \sum_{j=1}^{\infty} \prod_{i=1}^{2} \frac{1}{\mu\left(2^{j+1} B\right)} \int_{2^{j+1} B}\left|f_{i}\left(y_{i}\right)\right| d \mu\left(y_{i}\right) .
\end{aligned}
$$

From this, together with Hölder's inequality, (1.6) and Lemma 2.5, we deduce that

$$
\begin{aligned}
& \left|T\left(f_{1}^{\infty}, f_{2}^{\infty}\right)(x)\right| \\
\lesssim & \sum_{j=1}^{\infty} \prod_{i=1}^{2} \frac{1}{\mu\left(2^{j+1} B\right)} \int_{2^{j+1} B}\left|f_{i}\left(y_{i}\right)\right|\left[\omega_{i}\left(y_{i}\right)\right]^{1 / p_{i}}\left[\omega_{i}\left(y_{i}\right)\right]^{-1 / p_{i}} d \mu\left(y_{i}\right) \\
\leq & \sum_{j=1}^{\infty} \prod_{i=1}^{2} \frac{1}{\mu\left(2^{j+1} B\right)}\left[\left(\int_{2^{j+1} B}\left|f_{i}\left(y_{i}\right)\right|^{p_{i}} \omega_{i}\left(y_{i}\right) d \mu\left(y_{i}\right)\right)^{1 / p_{i}} \times\left(\int_{2^{j+1} B}\left[\omega_{i}\left(y_{i}\right)\right]^{1-p_{i}^{\prime}} d \mu\left(y_{i}\right)\right)^{1 / p_{i}^{\prime}}\right]
\end{aligned}
$$




$$
\begin{aligned}
& =\sum_{j=1}^{\infty} \frac{1}{\mu\left(2^{j+1} B\right)^{2}} \prod_{i=1}^{2}\left(\frac{1}{\mu\left(2^{j+1} B\right)} \int_{2^{j+1} B}\left[\omega_{i}\left(y_{i}\right)\right]^{1-p_{i}^{\prime}} d \mu\left(y_{i}\right)\right)^{1 / p_{i}^{\prime}} \times\left[\mu\left(2^{j+1} B\right)\right]^{1 / p_{i}^{\prime}} \\
& \quad \times\left(\frac{1}{\left[\omega_{i}\left(2^{j+1} B\right)\right]^{\kappa}} \int_{2^{j+1} B}\left|f_{i}\left(y_{i}\right)\right|^{p_{i}} \omega_{i}\left(y_{i}\right) d \mu\left(y_{i}\right)\right)^{1 / p_{i}} \times\left[\omega_{i}\left(2^{j+1} B\right)\right]^{\frac{\kappa}{p_{i}}} \\
& \leq \prod_{i=1}^{2}\left\|f_{i}\right\|_{M_{\omega_{i}}^{p_{i, k}}(\mathcal{X})} \cdot \sum_{j=1}^{\infty}\left(\frac{\prod_{i=1}^{2}\left[\omega_{i}\left(2^{j+1} B\right)\right]^{\kappa / p_{i}}}{\left[v_{\vec{\omega}}\left(2^{j+1} B\right)\right]^{1 / p}}\right) \\
& \lesssim \prod_{i=1}^{2}\left\|f_{i}\right\|_{M_{\omega_{i}}^{p_{i, k}}(\mathcal{X})} \cdot \sum_{j=1}^{\infty}\left[v_{\vec{\omega}}\left(2^{j+1} B\right)\right]^{(\kappa-1) / p} .
\end{aligned}
$$

Due to $v_{\vec{\omega}} \in A_{2 p}(\mu) \subset A_{\infty}(\mu)$ and Lemma 2.1, by taking $\delta=\frac{1}{2 p}$, we see that

$$
\frac{v_{\vec{\omega}}(B)}{v_{\vec{\omega}}\left(2^{j+1} B\right)} \lesssim\left(\frac{\mu(B)}{\mu\left(2^{j+1} B\right)}\right)^{\delta},
$$

which, together with (1.2), implies that

$$
\begin{aligned}
I_{4} & =\frac{1}{\left[v_{\vec{\omega}}(B)\right]^{\kappa / p}}\left(\int_{B}\left|T\left(f_{1}^{\infty}, f_{2}^{\infty}\right)(x)\right|^{p} v_{\vec{\omega}}(x) d \mu(x)\right)^{1 / p} \\
& \leq\left[v_{\vec{\omega}}(B)\right]^{(1-\kappa) / p} \sup _{x \in B}\left|T\left(f_{1}^{\infty}, f_{2}^{\infty}\right)(x)\right| \\
& \lesssim \prod_{i=1}^{2}\left\|f_{i}\right\|_{M_{\omega_{i}}^{p_{i}, \kappa}(\mathcal{X})} \cdot \sum_{j=1}^{\infty} \frac{\left[v_{\vec{\omega}}(B)\right]^{(1-\kappa) / p}}{\left[v_{\vec{\omega}}\left(2^{j+1} B\right)\right]^{(1-\kappa) / p}} \\
& \leq \prod_{i=1}^{2}\left\|f_{i}\right\|_{M_{\omega_{i}}^{p_{i}, \kappa}(\mathcal{X})} \cdot \sum_{j=1}^{\infty}\left(\frac{\mu(B)}{\mu\left(2^{j+1} B\right)}\right)^{\delta(1-\kappa) / p} \\
& \lesssim \prod_{i=1}^{2}\left\|f_{i}\right\|_{M_{\omega_{i}}^{p_{i}, \kappa}(\mathcal{X})} \cdot \sum_{j=1}^{\infty}\left(\frac{1}{2^{(j+1) \tau}}\right)^{\delta(1-\kappa) / p} \\
& \leq \prod_{i=1}^{2}\left\|f_{i}\right\|_{M_{\omega_{i}}^{p_{i}, \kappa}(\mathcal{X})^{\prime}}
\end{aligned}
$$

where the last inequality holds since $\kappa \in(0,1)$ and $\delta>0$.

We now consider the terms $I_{2}$ and $I_{3}$. We only give the arguments for $I_{2}$ since the arguments for $I_{3}$ are similar. Using the (1.3) again, we deduce that, for any $x \in B$,

$$
\begin{aligned}
\left|T\left(f_{1}^{\infty}, f_{2}^{0}\right)(x)\right| & =\left|\int_{\mathcal{X}^{2}} K\left(x, y_{1}, y_{2}\right) f_{1}^{\infty}\left(y_{1}\right) f_{2}^{0}\left(y_{2}\right) d \mu\left(y_{1}\right) d \mu\left(y_{2}\right)\right| \\
& \lesssim \int_{\mathcal{X}^{2}} \frac{\left|f_{1}^{\infty}\left(y_{1}\right) f_{2}^{0}\left(y_{2}\right)\right|}{\left[\sum_{i=1}^{2} V\left(x, y_{i}\right)\right]^{2}} d \mu\left(y_{1}\right) d \mu\left(y_{2}\right)
\end{aligned}
$$




$$
\begin{aligned}
& \leq \int_{2 B}\left|f_{2}\left(y_{2}\right)\right| d y_{2} \times \int_{\mathcal{X} \backslash(2 B)} \frac{\left|f_{1}\left(y_{1}\right)\right|}{\left[V\left(x, y_{1}\right)\right]^{2}} d \mu\left(y_{1}\right) \\
& \lesssim \int_{2 B}\left|f_{2}\left(y_{2}\right)\right| d \mu\left(y_{2}\right) \times \sum_{j=1}^{\infty} \int_{\left(2^{j+1} B \backslash 2^{j} B\right)} \frac{\left|f_{1}\left(y_{1}\right)\right|}{\left[V\left(x, y_{1}\right)\right]^{2}} d \mu\left(y_{1}\right) \\
& \lesssim \int_{2 B}\left|f_{2}\left(y_{2}\right)\right| d \mu\left(y_{2}\right) \times \sum_{j=1}^{\infty} \frac{1}{\mu\left(2^{j+1} B\right)^{2}} \int_{2^{j+1} B \backslash 2^{j} B}\left|f_{1}\left(y_{1}\right)\right| d \mu\left(y_{1}\right) \\
& \leq \sum_{j=1}^{\infty} \prod_{i=1}^{2} \frac{1}{\mu\left(2^{j+1} B\right)} \int_{2^{j+1} B}\left|f_{i}\left(y_{i}\right)\right| d \mu\left(y_{i}\right) .
\end{aligned}
$$

It then follow from (3.1) that

$$
\left|T\left(f_{1}^{\infty}, f_{2}^{0}\right)(x)\right| \lesssim \prod_{i=1}^{2}\left\|f_{i}\right\|_{M_{\omega_{i}}^{p_{j}, \kappa}(\mathcal{X})} \cdot \sum_{j=1}^{\infty}\left[v_{\vec{\omega}}\left(2^{j+1} B\right)\right]^{(\kappa-1) / p}
$$

Similar to (3.2), we have

$$
\begin{aligned}
I_{2} & \leq\left[v_{\vec{\omega}}(B)\right]^{(1-\kappa) / p} \sup _{x \in B}\left|T\left(f_{1}^{\infty}, f_{2}^{0}\right)(x)\right| \\
& \lesssim \prod_{i=1}^{2}\left\|f_{i}\right\|_{M_{\omega_{i}}^{p_{i}, \kappa}(\mathcal{X})} \cdot \sum_{j=1}^{\infty} \frac{\left[v_{\vec{\omega}}(B)\right]^{(1-\kappa) / p}}{\left[v_{\vec{\omega}}\left(2^{j+1} B\right)\right]^{(1-\kappa) / p}} \\
& \lesssim \prod_{i=1}^{2}\left\|f_{i}\right\|_{M_{\omega_{i}}^{p_{j}, \kappa}(\mathcal{X})}
\end{aligned}
$$

Combining the above estimates and then taking the supremum over all balls $B \subset \mathcal{X}$, we finish the proof of Theorem 1.1.

Proof of Theorem 1.2. For any ball $B=B\left(c_{B}, r_{B}\right)$, decompose $f_{i}=f_{i}^{0}+f_{i}^{\infty}$ with the same notation in the proof of Theorem 1.1. Recall that

$$
\begin{aligned}
\prod_{i=1}^{2} f_{i}\left(y_{i}\right) & =\prod_{i=1}^{2}\left(f_{i}^{0}\left(y_{i}\right)+f_{i}^{\infty}\left(y_{i}\right)\right) \\
& =f_{1}^{0}\left(y_{1}\right) f_{2}^{0}\left(y_{2}\right)+f_{1}^{0}\left(y_{1}\right) f_{2}^{\infty}\left(y_{2}\right)+f_{1}^{\infty}\left(y_{1}\right) f_{2}^{0}\left(y_{2}\right)+f_{1}^{\infty}\left(y_{1}\right) f_{2}^{\infty}\left(y_{2}\right) .
\end{aligned}
$$

Then we have, for any $\lambda \in(0, \infty)$,

$$
\begin{aligned}
& v_{\vec{\omega}}\left(\left\{x \in B:\left|T\left(f_{1}, f_{2}\right)(x)\right|>\lambda\right\}\right)^{1 / p} \\
& \lesssim v_{\vec{\omega}}\left(\left\{x \in B:\left|T\left(f_{1}^{0}, f_{2}^{0}\right)\right|>\frac{\lambda}{4}\right\}\right)^{1 / p}+v_{\vec{\omega}}\left(\left\{x \in B:\left|T\left(f_{1}^{0}, f_{2}^{\infty}\right)\right|>\frac{\lambda}{4}\right\}\right)^{1 / p} \\
& \quad+v_{\vec{\omega}}\left(\left\{x \in B:\left|T\left(f_{1}^{\infty}, f_{2}^{0}\right)\right|>\frac{\lambda}{4}\right\}\right)^{1 / p}+v_{\vec{\omega}}\left(\left\{x \in B:\left|T\left(f_{1}^{\infty}, f_{2}^{\infty}\right)\right|>\frac{\lambda}{4}\right\}\right)^{1 / p} \\
& =: \\
& I_{1}^{*}+I_{2}^{*}+I_{3}^{*}+I_{4}^{*} .
\end{aligned}
$$


By Lemma 2.2 again, we know that $v_{\vec{\omega}} \in A_{2 p}(\mu)$ with $2 p \in[1, \infty)$. Applying Lemmas 2.6, 2.5 and 2.1, we have

$$
\begin{aligned}
I_{1}^{*} & \lesssim \frac{1}{\lambda} \prod_{i=1}^{2}\left(\int_{2 B}\left|f_{i}(x)\right|^{p_{i}} \omega_{i}(x) d \mu(x)\right)^{1 / p_{i}} \\
& =\frac{1}{\lambda} \prod_{i=1}^{2}\left[\frac{\left.\omega_{i}(2 B)\right]^{\kappa / p_{i}}}{\left[\omega_{i}(2 B)\right]^{\kappa / p_{i}}}\left(\int_{2 B}\left|f_{i}\left(x_{i}\right)\right|^{p_{i}} \omega_{i}(x) d \mu(x)\right)^{1 / p_{i}}\right. \\
& \lesssim \frac{\prod_{i=1}^{2}\left[\omega_{i}(2 B)\right]^{\kappa / p_{i}}}{\lambda} \cdot \prod_{i=1}^{2}\left\|f_{i}\right\|_{M_{\omega_{i}}^{p_{i}, \kappa}(\mathcal{X})} \\
& \leq \frac{\left[v_{\vec{\omega}}(2 B)\right]^{\kappa / p}}{\lambda} \prod_{i=1}^{2}\left\|f_{i}\right\|_{M_{\omega_{i}}^{p_{i}, \kappa}(\mathcal{X})} \\
& \lesssim \frac{\left[v_{\vec{\omega}}(B)\right]^{\kappa / p}}{\lambda} \prod_{i=1}^{2}\left\|f_{i}\right\|_{M_{\omega_{i}}^{p_{i}, \kappa}(\mathcal{X})} .
\end{aligned}
$$

In the proof of Theorem 1.1, for $\left(\alpha_{1}, \alpha_{2}\right)=(\infty, \infty)$ or $(0, \infty)$ or $(0, \infty)$, we have already showed the following pointwise estimate: for any $x \in B$,

$$
\left|T\left(f_{1}^{\alpha_{1}}, f_{2}^{\alpha_{2}}\right)(x)\right| \lesssim \sum_{j=1}^{\infty} \prod_{i=1}^{2} \frac{1}{\mu\left(2^{j+1} B\right)} \int_{2^{j+1} B}\left|f_{i}\left(y_{i}\right)\right| d \mu\left(y_{i}\right) .
$$

Without loss of generality, we assume that $p_{1}=1$ and $p_{2}>1$, then we have

$$
\begin{aligned}
& \left|T\left(f_{1}^{\alpha_{1}}, f_{2}^{\alpha_{2}}\right)(x)\right| \\
\lesssim & \sum_{j=1}^{\infty}\left\{\frac{1}{\mu\left(2^{j+1} B\right)} \int_{2^{j+1} B}\left|f_{1}\left(y_{1}\right)\right| d \mu\left(y_{1}\right) \times \frac{1}{\mu\left(2^{j+1} B\right)} \int_{2^{j+1} B}\left|f_{2}\left(y_{2}\right)\right| d \mu\left(y_{2}\right)\right\} \\
\leq & \sum_{j=1}^{\infty}\left\{\frac{1}{\mu\left(2^{j+1} B\right)} \int_{2^{j+1} B}\left|f_{1}\left(y_{1}\right)\right| \omega_{1}\left(y_{1}\right) d \mu\left(y_{1}\right) \frac{1}{\inf _{2^{j+1} B} \omega_{1}}\right. \\
& \left.\times \frac{1}{\mu\left(2^{j+1} B\right)} \int_{2^{j+1} B}\left|f_{2}\left(y_{2}\right)\right|\left[\omega_{2}\left(y_{2}\right)\right]^{\frac{1}{p_{2}}}\left[\omega_{i}\left(y_{i}\right)\right]^{\frac{-1}{p_{2}}} d \mu\left(y_{2}\right)\right\} \\
\lesssim & \prod_{i=1}^{2}\left\|f_{i}\right\|_{M_{\omega_{i}}^{p_{i}, \kappa}(\mathcal{X})} \sum_{j=1}^{\infty}\left[v_{\vec{\omega}}\left(2^{j+1} B\right)\right]^{(\kappa-1) / p} .
\end{aligned}
$$

Similar to (3.2), we have, for any $x \in B$,

$$
\begin{aligned}
& \left|T\left(f_{1}^{\alpha_{1}}, f_{2}^{\alpha_{2}}\right)(x)\right| \\
\lesssim & \prod_{i=1}^{2}\left\|f_{i}\right\|_{M_{\omega_{i}}^{p_{i}, \kappa}(\mathcal{X})} \cdot \frac{1}{\left[v_{\vec{\omega}}(B)\right]^{(1-\kappa) / p}} \sum_{j=1}^{\infty}\left[\frac{\left[v_{\vec{\omega}}(B)\right]^{(1-\kappa) / p}}{\left[v_{\vec{\omega}}\left(2^{j+1} B\right)\right]^{(1-\kappa) / p}}\right]
\end{aligned}
$$




$$
\begin{aligned}
& \lesssim \prod_{i=1}^{2}\left\|f_{i}\right\|_{M_{\omega_{i}}^{p_{j}, \mathcal{X}}(\mathcal{X})} \cdot \frac{1}{\left[v_{\vec{\omega}}(B)\right]^{(1-\kappa) / p}} \sum_{j=1}^{\infty}\left[\left(\frac{\mu(B)}{\mu\left(2^{j+1} B\right)}\right)^{\delta(1-\kappa) / p}\right] \\
& \lesssim \prod_{i=1}^{2}\left\|f_{i}\right\|_{M_{\omega_{i}}^{p_{j}, \mathcal{X}}(\mathcal{X})} \cdot \frac{1}{\left[v_{\vec{\omega}}(B)\right]^{(1-\kappa) / p}} .
\end{aligned}
$$

If $\left\{x \in B:\left|T\left(f_{1}^{\alpha_{1}}, f_{2}^{\alpha_{2}}\right)(x)\right|>\lambda / 4\right\}=\varnothing$, then the inequality

$$
I_{j}^{*}=v_{\vec{\omega}}\left(\left\{x \in B:\left|T\left(f_{1}^{\alpha_{1}}, f_{2}^{\alpha_{2}}\right)(x)\right|>\frac{\lambda}{4}\right\}\right)^{1 / p} \lesssim \frac{\left[v_{\vec{\omega}}(B)\right]^{\kappa / p}}{\lambda} \prod_{i=1}^{2}\left\|f_{i}\right\|_{M_{\omega_{i}}^{p_{i}, \kappa}(\mathcal{X})}
$$

holds trivially for $j=2,3,4$.

Now, if $\left\{x \in B:\left|T\left(f_{1}^{\alpha_{1}}, f_{2}^{\alpha_{2}}\right)(x)\right|>\lambda / 4\right\} \neq \varnothing$, then, by the inequality (3.3), we have

$$
\lambda \lesssim \prod_{i=1}^{2}\left\|f_{i}\right\|_{M_{\omega_{i}}^{p_{i} \kappa}(\mathcal{X})} \cdot \frac{1}{\left[v_{\vec{\omega}}(B)\right]^{(1-\kappa) / p}}
$$

which is equivalent to

$$
\left[v_{\vec{\omega}}(B)\right]^{1 / p} \lesssim \frac{\left[v_{\vec{\omega}}(B)\right]^{\kappa / p}}{\lambda} \prod_{i=1}^{m}\left\|f_{i}\right\|_{M_{\omega_{i}}^{p_{i}, \kappa}(\mathcal{X})} .
$$

Therefore, for $j=2,3,4$,

$$
I_{j}^{*} \leq\left[v_{\vec{\omega}}(B)\right]^{1 / p} \lesssim \frac{\left[v_{\vec{\omega}}(B)\right]^{\kappa / p}}{\lambda} \prod_{i=1}^{2}\left\|f_{i}\right\|_{M_{\omega_{i}}^{p_{i}, \kappa}(\mathcal{X})^{\prime}}
$$

which completes the proof of Theorem 1.2.

\section{Acknowledgements}

The authors would like to thank the referees for their time and comments. This work is supported by the National Natural Science Foundation of China (No. 12071052).

\section{References}

[1] D. Chen, S. Lu and S. Mao, Multiple weighted estimates for maximal vector-valued commutator of multilinear Calderón-Zygmund singular integrals, Front. Math. China, 12(3) (2017), 531-558.

[2] M. Cheng, D. Deng and R. Long, Real Analysis (in Chinese), Second Edition, Higher Education Press, Beijing, 2008.

[3] J. Chou, X. Li, Y. Tong and H. Lin, Generalized weighted Morrey spaces on RD-spaces, Rocky Mountain J. Math., 50(4) (2020), 1277-1293. 
[4] R. R. Coifman and Y. Meyer, On commutators of singular integrals and bilinear singular integrals, Trans. Amer. Math. Soc., 212 (1975), 315-331.

[5] R. R. Coifman and G. Weiss, Analyse Harmonique Non-commutative sur Certains Espaces Homogénes, Étude de certaines intégrales singulières, Lecture Notes in Math., 242, SpringerVerlag, Berlin-New York, 1971.

[6] R. R. Coifman and G. Weiss, Extensions of Hardy spaces and their use in analysis, Bull. Amer. Math. Soc., 83 (1977), 569-645.

[7] L. Grafakos, Modern Fourier Analysis, Second Edition, Grad. Texts in Math., 250, Springer, New York, 2009.

[8] L. Grafakos, L. Liu, S. Lu and F. Zhao, The multilinear Marcinkiewicz interpolation theorem revisited: The behavior of the constant, J. Funct. Anal., 262(5) (2012), 2289-2313.

[9] L. Grafakos, L. Liu, D. Maldonedo and D. Yang, Multilinear analysis on metric spaces, Dissertationes Math., 497 (2014), 1-121.

[10] L. Grafakos and R. H. Torres, Multilinear Calderón-Zygmund theory, Adv. Math., 165(1) (2002), 124-164.

[11] L. Grafakos and R. H. Torres, Maximal operator and weighted norm inequalities for multilinear singular integrals, Indiana Univ. Math. J., 51(5) (2002), 1261-1276.

[12] Y. Han, D. Müller and D. Yang, A theory of Besov and Triebel-Lizorkin spaces on metric measure spaces modeled on Carnot-Carathédory spaces, Abstr. Appl. Anal., (2008), Art. ID 893409.

[13] G. Hu and S. Lu, Weighted estimates for the multilinear singular integral operators with non-smooth kernels, Sci. China Math., 54(3) (2011), 587-602.

[14] V. Kokilashvili and A. Meskhi, The boundedness of sublinear operators in weighted Morrey spaces defined on spaces of homogeneous type, pp. 193-211 in Function Spaces and Inequalities, Springer Proc. Math. Stat., 206, Springer, 2017.

[15] Y. Komori and S. Shirai, Weighted Morrey space and a singular integral operator, Math. Nachr., 282(2) (2009), 219-231.

[16] A. K. Lerner, S. Ombrosi, C. Pérez, R. H. Torres, and R. Trujillo-González, New maximal functions and multiple weights for the multilinear Calderón-Zygmund theory, Adv. Math., 220(4) (2009), 1222-1264.

[17] Y. Lin, G. Lu and S. Lu, Sharp maximal estimates for multilinear commutators of multilinear strongly singular Calderón-Zygmund operators and applications, Forum Math., 31(1) (2019), 1-18.

[18] H. Mo and S. Lu, Commutators generated by multilinear Calderón-Zygmund type singular integral and Lipschitz functions, Acta Math. Appl. Sin. Engl. Ser., 30(4) (2014), 903-912.

[19] C. B. Morrey, On the solutions of quasi-linear elliptic partial differential equations, Trans. Amer. Math. Soc., 43(1) (1938), 126-126.

[20] D. Müller and D. Yang, A difference characterization of Besov and Triebel-Lizorkin spaces on RD-spaces, Forum Math., 21(2) (2009), 259-298.

[21] J. O. Strömberg and A. Torchinsky, Weighted Hardy Spaces, Lecture Notes in Mathematics, 1381, Springer-Verlag, Berlin, 1989.

[22] H. Wang and W. Yi, Multilinear singular and fractional integral operators on weighted Morrey spaces, J. Funct. Spaces Appl., (2013), Art. ID 735795.

[23] D. Yang and Y. Zhou, Radial maximal function characterizations of Hardy spaces on RDspaces and their applications, Math. Ann., 346(2) (2010), 307-333.

[24] D. Yang, Y. Zhou, New properties of Besov and Triebel-Lizorkin spaces on RD-spaces, Manuscripta Math., 134(1-2) (2011), 59-90. 
[25] D. Yang and Y. Zhou, Localized Hardy spaces $H^{1}$ related to admissible functions on RDspaces and applications to Schrödinger operators, Trans. Amer. Math. Soc., 363(3) (2011), 1197-1239. 